\title{
T-type calcium channels in the orbitofrontal cortex mediate sensory integration as measured using a spontaneous oddity task in rats
}

\author{
Wendie N. Marks, ${ }^{1}$ Madeline E. Parker, ${ }^{1}$ Nadine K. Zabder, ${ }^{1}$ Quentin Greba, ${ }^{1}$ \\ Terrance P. Snutch, ${ }^{2}$ and John G. Howland ${ }^{1}$ \\ ${ }^{1}$ Department of Physiology, University of Saskatchewan, Saskatoon, Saskatchewan S7N 5E5, Canada; ${ }^{2}$ Michael Smith Laboratories \\ and Djavad Mowafaghian Centre for Brain Health, University of British Columbia, Vancouver, British Columbia V6T 1Z4, Canada
}

\begin{abstract}
The roles of low-voltage-activated (T-type) calcium channels in brain diseases have been studied extensively. Less is known regarding the involvement of T-type channels in cognition and behavior. Sensory integration (SI) is a cognitive process whereby the brain uses unimodal or multimodal sensory features to create a comprehensive representation of the environment. The multisensory object oddity (MSO) task assesses SI using combinations of sensory features of objects, either in the same or different sensory modalities. The regulation of SI involves the orbitofrontal cortex (OFC), an area which shows high levels of T-type calcium channel expression. We tested the effects of blocking T-type calcium channels on the MSO task with the selective T-type antagonist, Z944 (5 mg/ kg; i.p. systemic; 100 or $500 \mu \mathrm{M}$ OFC infusion), in male Long Evans rats. With systemic treatment, Z944 impaired the visual and visual-olfactory versions of the task. Infusion of 100 and $500 \mu \mathrm{M} \mathrm{Z944}$ produced deficits in the olfactory version of the task. In addition, only vehicle-infused, but not Z944-infused, rats showed significant performance above chance for all task variants. Thus, the present results suggest that T-type calcium channels in OFC are involved in SI of features in an oddity task. Given that unimodal SI was disrupted by OFC infusions of Z944, the deficits in the multimodal task must be interpreted with caution. As SI is disrupted in psychiatric disorders, further investigations elucidating the brain regions implicated in SI regulation by T-type calcium channels may help inform therapeutic development for those suffering from SI impairments.
\end{abstract}

Processing sensory information into contextually rich representations of the environment is fundamental to cognition. The ability of the brain to generate a coherent representation of reality by consolidating simultaneously experienced stimuli from the same or different modalities is termed sensory integration (SI) or multisensory integration (MSI), respectively (Stein and Stanford 2008; Cloke et al. 2016). Cognitive abnormalities are characteristic symptoms of brain disorders such as schizophrenia, autism, and epilepsy (Caplan et al. 2008; Keefe and Harvey 2012; Leekam 2016). Some researchers have hypothesized that deficits in complex cognitive functions, such as the sociability deficits observed in autism, may be the result of impairments in lower-level cognitive processes such as MSI (Baum et al. 2015). Indeed, MSI impairments are observed in neuropsychiatric populations (Oberman and Ramachandran 2008; Tseng et al. 2015) and impaired SI of unimodal stimuli is also observed during some symptoms such as psychosis (Carter et al. 2017). Despite the known deficits in MSI in human psychiatric populations, progress in this area has been hindered by a lack of valid behavioral assessments for animal models, particularly rodents (Cloke et al. 2016). Considering the severity of cognitive symptoms in psychiatric illness correlate strongly with patient functional outcome (Tamminga et al. 1998), the need for additional insight into the neural bases of MSI in both healthy and disease states is needed.

Recent progress has been made in assessing MSI in rodents with the development of a tactile-to-visual cross-modal object recognition (CMOR) task (Winters and Reid 2010). However,

\section{Corresponding author: john.howland@usask.ca}

Article is online at http://www.learnmem.org/cgi/doi/10.1101//m.047332. 118. aspects of CMOR's design prevent a direct examination of this cognitive process. In particular, CMOR cannot assess MSI in the absence of mnemonic demand as there is a delay between an initial object exploration phase and object recognition phase that requires memory. A novel paradigm, the multisensory object oddity (MSO) task, improves upon the limitation of CMOR in this regard (Cloke et al. 2016). Similar to CMOR and other spontaneous object recognition tasks, MSO exploits rodents' innate tendency to explore oddity and is advantageous in that it provides a nonaversive means of assessing MSI that does not require prior training (Winters et al. 2008). However, in contrast to CMOR, the MSO lacks a mnemonic requirement as MSO is a spontaneous exploration task without a delay. Furthermore, MSO improves upon CMOR by increasing the translatability of results through the simultaneous presentation of modality-specific features, analogous to human MSI paradigms (Cloke et al. 2016).

T-type calcium channels are a class of voltage-gated calcium channels (VGCCs), which activate near resting potentials. These low-threshold channels are expressed widely in the brain and their roles in nociception and epilepsy have been studied extensively, whereas comparatively little is known regarding their contribution to cognition and behavior. Recent evidence from our laboratory suggests T-type calcium channels are involved in the normal expression of MSI. We previously examined the effects of systemic administration of Z944, a highly selective pan-T-type calcium

(C) 2018 Marks et al. This article is distributed exclusively by Cold Spring Harbor Laboratory Press for the first 12 months after the full-issue publication date (see http://learnmem.cshlp.org/site/misc/terms.xhtml). After 12 months, it is available under a Creative Commons License (Attribution-NonCommercial 4.0 International), as described at http://creativecommons.org/licenses/by-nc/4.0/. 
channel antagonist, on CMOR in two rat strains, a rat model of childhood absence epilepsy, the genetic absence epilepsy rats from Strasbourg (GAERS), and a nonepileptic control (NEC) strain (Marks et al. 2016a). Similar to childhood absence epilepsy patients, GAERS demonstrate psychiatric-like phenotypes and notably possess a hypermorphic missense mutation in the Cav3.2 T-type calcium channel gene linked to thalamocortical hyperexcitability implicated in seizure generation and propagation (Jones et al. 2008, 2010; Tringham et al. 2012; Marks et al. 2016b). Treatment with Z944 reversed cross-modal and visual memory deficits on CMOR in GAERS, while impairments were induced in NECs, suggesting an optimal amount of T-type calcium channel activity is required for both uni- and multimodal memory (Marks et al. 2016a). An emerging body of genetic evidence implicates aberrant T-type calcium channel activity in the etiology of neuropsychiatric disorders other than epilepsy. Genome-wide association studies have revealed that missense mutations in the Cav3.3 and Cav3.2 T-type calcium channel genes occur with an increased frequency in individuals with schizophrenia and autism, respectively (Splawski et al. 2006; Andrade et al. 2016). Along with our CMOR data, these studies indicate that further characterizing the involvement of T-type calcium channels in MSI is essential to our understanding of this cognitive function and its disruption in neurological and psychiatric disorders.

To explore the role of T-type calcium channels in MSI, we examined the effects of Z944 on MSO performance when administered both systemically and into the orbitofrontal cortex (OFC), a region in which T-type calcium channels are expressed (Talley et al. 1999). Multiple lines of experimental evidence suggest the OFC plays a critical role in the distributed network of cortical and subcortical brain regions thought to subserve MSI (Cloke and Winters 2015). Cytoarchitectural studies indicate the OFC receives convergent visual, somatosensory, olfactory, and gustatory inputs in both rats and nonhuman primates (Ongür and Price 2000). Bilateral excitotoxic OFC lesions were shown to induce significant cross-modal memory deficits on CMOR without impacting tactile or visual memory, suggesting the OFC participates in multimodal, but not unimodal, perception (Reid et al. 2014). Similarly, aspiration lesions of the OFC selectively impaired a tactile-olfactory discrimination task while olfactory processing remained intact (Whishaw et al. 1992). Recent MSO data suggests the OFC participates in multimodal perception in the absence of mnemonic demand as well (Cloke et al. 2016). Therefore, we hypothesized that intraorbitofrontal infusion of Z944 would dosedependently impair MSO performance while leaving unimodal perception intact. In contrast, systemic administration of Z944 was postulated to attenuate multimodal and unimodal visual oddity, due to the presence of T-type calcium channels in brain regions associated with both processes (Talley et al. 1999; Winters and Reid 2010).

\section{Results}

An initial cohort of rats $(n=8)$ was tested to confirm significant exploration of the odd object in treatment naïve rats for each version of the task (Fig. 1). A one-sample $t$-test comparing oddity preference to chance performance (Table 1) confirmed significant oddity preference for each task variant (visual: $t_{(7)}=2.40$, $P=0.048$; olfactory: $t_{(7)}=4.35, P=0.003$; visual-olfactory: $t_{(7)}=7.14$, $P<0.001)$.

\section{Systemic Z944 administration}

The effect of Z944 treatment (5 mg/kg; intraperitoneal) on the total amount of time spent exploring all objects for each task was examined (Table 2). The effect of Z944 on object exploration

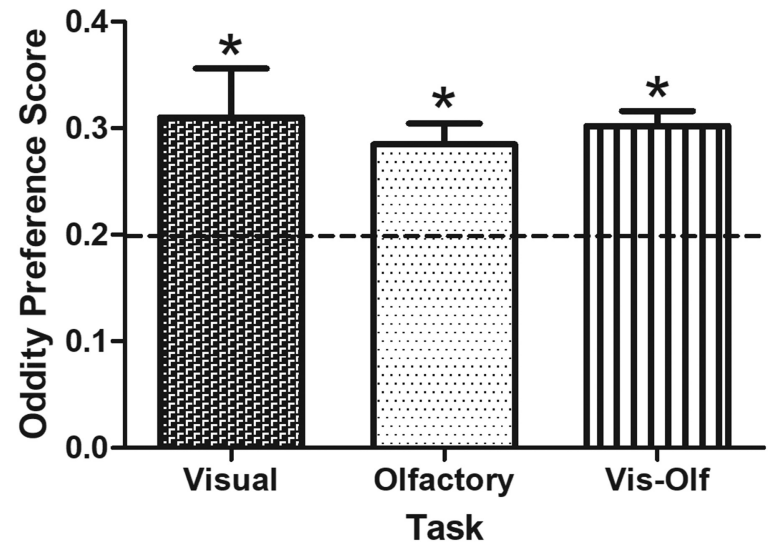

Figure 1. Visual, olfactory, and visual-olfactory oddity preference for drug naïve rats. Rats showed significant task performance for all three task variants $\left(^{*}\right) P<0.05$. A mean of 0.2 represents chance performance.

was not significant for any of the tasks (all $P \geq 0.057$ ). Performance in each task was examined for the effects of treatment and time bin (1 min intervals). The main effect of all Time Bin and Time Bin by Treatment interactions were nonsignificant (all $P \geq 0.077$ ). A main effect of Treatment was found for the visual (Fig. $2 \mathrm{~A} ; F_{(1,19)}=5.99$, $P=0.024$, partial $\left.\eta^{2}=0.240\right)$, and visual-olfactory task $\left(F_{(1,26)}=\right.$ 4.44, $P=0.045$, partial $\left.\eta^{2}=0.146\right)$. Z944 significantly decreased oddity preference for both tasks. One-sample $t$-tests comparing the oddity exploration of each treatment group during each task to chance performance (Table 1) showed significant oddity preference when rats received vehicle treatment for all tasks (visual: $t_{(19)}=$ 3.70, $P=0.002$; olfactory: $t_{(27)}=3.14, P=0.004$; visual-olfactory: $\left.t_{(26)}=3.07, P=0.005\right)$. Rats also showed significant oddity preference during the olfactory task when treated with Z944 $\left(t_{(27)}=2.62\right.$, $P=0.014)$.

\section{Z944 infusion into the orbitofrontal cortex}

Similar to systemic treatment with Z944, bilateral infusion of Z944 into the OFC did not significantly affect the amount of time spent exploring the objects for any of the task variants $(P \geq 0.090$, see Table 2). Repeated measures analyses examining the effects of treatment and time bin on oddity preference revealed nonsignificant main effects of both Time Bin and Time Bin by Treatment interactions for all tasks (all $P \geq 0.173$ ). The number of infusions a rat had sustained also did not affect oddity preference (ANOVA, $P>0.082)$. However, a significant main effect of Treatment was found for the olfactory task (Fig. $2 \mathrm{~B} ; F_{(2,16)}=4.61, P=0.026$, partial $\left.\eta^{2}=0.365\right)$. Post-hoc analyses revealed a significant decrease in oddity preference in the $100 \mu \mathrm{M}\left(F_{(1,8)}=45.16, P<0.001\right)$ and $500 \mu \mathrm{M}$ concentrations $\left(F_{(1,8)}=8.03, P=0.022\right)$. One-sample $t$-tests comparing oddity preference to chance performance for each treatment group (Table 1) revealed significant oddity preference when rats received vehicle treatment during all tasks (visual: $t_{(8)}=2.66$, $P=0.029$; olfactory: $t_{(8)}=3.17, P=0.013$, visual-olfactory: $t_{(20)}=$ $2.36, P=0.028$ ).

\section{Discussion}

In this study, we examined the effects of the T-type calcium channel blocker, Z944, on performance of the MSO task, a novel behavioral measure of unimodal and multimodal SI. Treatment naïve rats performed the visual, olfactory, and, visual-olfactory MSO tasks significantly above chance (Fig. 1). Systemic treatment with $5 \mathrm{mg} / \mathrm{kg}$ of Z944 significantly impaired unimodal visual and 
T-type calcium cannels mediate sensory integration

Table 1. Oddity preference group means and one-sample $t$-test values (mean oddity preference; $t$-test value) comparing group means to chance performance (a mean of 0.2 represents chance performance)

\begin{tabular}{|c|c|c|c|c|}
\hline Administration & Treatment & Visual & Olfactory & Visual-Olfactory \\
\hline & No treatment & $0.31 ; 2.40^{*}$ & $0.28 ; 4.35^{\star \star}$ & $0.30 ; 7.13^{\star \star *}$ \\
\hline \multirow[t]{2}{*}{ Systemic } & Vehicle & $0.29 ; 3.70^{* *}$ & $0.27 ; 3.14^{\star \star}$ & $0.25 ; 3.07^{\star *}$ \\
\hline & 5 mg/kg Z944 & $0.21 ; 0.35$ & $0.26 ; 2.62^{*}$ & $0.19 ;-0.48$ \\
\hline \multirow[t]{3}{*}{ Infusion } & Vehicle & $0.29 ; 2.66^{\star}$ & $0.25 ; 3.17^{\star}$ & $0.23 ; 2.36^{\star}$ \\
\hline & $100 \mu \mathrm{M}$ Z944 & $0.26 ; 1.72$ & $0.19 ;-1.16$ & $0.21 ; 0.28$ \\
\hline & $500 \mu \mathrm{M} Z 944$ & $0.24 ; 0.88$ & $0.20 ;-0.34$ & $0.20 ; 0.07$ \\
\hline
\end{tabular}

*significant at $P \leq 0.05 ;{ }^{* *}$ significant at $P \leq 0.01 ;{ }^{* * *}$ significant at $P \leq 0.001$.

multimodal visual-olfactory performance while leaving unimodal olfactory performance intact (Fig. 2A). In addition, infusion of Z944 into the OFC robustly impaired unimodal olfactory performance (Fig. 2B). Visual and multimodal visual-olfactory task performance were also impaired by the infusion of Z944 into the $\mathrm{OFC}$ as reflected by the failure of these rats to successfully perform these tasks, although between group differences did not reach significance (Fig. 2B). It is worth noting that the oddity preferences on the MSO task we observed for vehicle-treated rats are not as robust as those observed by Cloke et al. (2016), which may be explained by factors such as variability in environmental conditions between laboratories or differences in testing methodologies. Based on these results, we conclude that T-type calcium channels in the OFC are integrally involved in unimodal SI and potentially MSI.

\section{Systemic administration of Z944 disrupts unimodal visual, and multimodal visual-olfactory MSO task performance}

As hypothesized, visual and visual-olfactory task deficits in MSO produced by systemic Z944 treatment were found, an effect similar to the CMOR deficits previously reported by our laboratory. GAERS and Z944-treated NEC rats showed unimodal visual as well as visual-tactile CMOR deficits (Marks et al. 2016a). GAERS demonstrate a gain-in-function of Cav3.2 T-type calcium channel activity (Powell et al. 2009). Considering Z944 reverses CMOR impairments in GAERS, but produces deficits in NEC and Long Evans rats, an optimal amount of T-type calcium channel activation is likely required for unimodal visual and multimodal SI (Marks et al. 2016a). Importantly, the current study showed that uniand multimodal sensory deficits occur in the absence of the mnemonic demands required for CMOR performance (Winters and Reid 2010). Thus, the current work expands on the understanding of the role of T-type calcium channels in cognition in that T-type calcium channel antagonism disrupts not only sensory-specific object recognition memory, but also SI-specific processes. Further, deficits were found for visual-olfactory MSO demonstrating that the effects of Z944 on MSI extend beyond the visual-tactile MSI deficits found in CMOR (Marks et al. 2016a). The effect of systemic Z944 may be mediated by the PRh as the PRh is particularly important for visual recognition (Bartko et al. 2007a,b; Winters and Reid 2010), and has abundant T-type calcium channel expression (Talley et al. 1999). The occipital and temporal areas that comprise the ventral visual processing stream may also mediate the effects of Z944 as they are critical to object recognition and express T-type calcium channels (Talley et al. 1999; DiCarlo et al. 2012). Indeed, a lack of effect of systemic Z944 treatment on olfactory MSO suggests that systemic Z944 administration effects may be specific to visual sensory processing. From these findings, we conclude that unimodal visual and multimodal visual dependent task performance are particularly sensitive to T-type calcium channel activity.

The effects of systemic Z944 treatment on MSI are similar to those observed following NMDA receptor antagonist administration (Jacklin et al. 2012; Cloke and Winters 2015; Cloke et al. 2016). However, impaired unimodal SI was not found in these studies except when the task included a $1 \mathrm{~h}$ delay (Jacklin et al. 2012; Cloke and Winters 2015). Maternal immune activation offspring showed intact unimodal CMOR task performance with a $1 \mathrm{~h}$ delay while performance was impaired in the cross-modal portion of the task (Ballendine et al. 2015). Interestingly, we previously observed preserved recognition memory in a short-delay visual CMOR task in rats following systemic treatment with Z944 (Marks et al. 2016a). The lack of effect of Z944 on the short-delay visual CMOR versus the visual MSO task could be explained by fundamental differences in the cognitive demands of the respective tasks. The short-delay visual CMOR task depends on twochoice discrimination of visually distinct objects (Winters and Reid 2010). Alternatively, the cognitive demands of the visual MSO task are arguably greater in that the task can only be solved by using a conjunctive representation of the distinct visual features of the MSO object pairs (Bartko et al. 2007b; Cloke et al. 2016). Previous researchers have examined the effects of PRh lesions on both zero-delay two-choice visual object discrimination and on visual oddity that depends on the resolution of configural feature ambiguity (Bartko et al. 2007a,b). Indeed, it was found that PRh lesions did not impair performance of a zero-delay visual object discrimination task when the objects had a high level of visual distinction (Bartko et al. 2007a), a task similar to short-delay visual CMOR. Conversely, PRh lesions did impair performance of both a visual minimal-delay configural object recognition task and a visual configural oddity discrimination task (Bartko et al. 2007b), tasks similar to visual MSO. Thus, the PRh appears critical for task performance when the mnemonic demand of the task is high, such as with a $1 \mathrm{~h}$ delay visual CMOR task (Winters and Reid 2010), but also when the task relies on a conjunctive representation

Table 2. Total exploration time of the objects $(s \pm$ SEM) during testing

\begin{tabular}{llrrr}
\hline Administration & Treatment & Visual & Olfactory & Visual-Olfactory \\
\hline Systemic & Vehicle & $7.13 \pm 0.79$ & $13.47 \pm 1.15$ & $17.71 \pm 1.32$ \\
Infusion & $5 \mathrm{mg} / \mathrm{kg}$ Z944 & $8.63 \pm 0.62$ & $11.82 \pm 1.40$ & $16.63 \pm 1.64$ \\
& Vehicle & $6.43 \pm 0.92$ & $8.12 \pm 1.21$ & $13.27 \pm 1.00$ \\
& $100 \mu \mathrm{M}$ Z944 & $9.23 \pm 1.57$ & $7.82 \pm 1.02$ & $1.83 \pm 1.33$ \\
& $500 \mu \mathrm{M}$ Z944 & $9.69 \pm 0.77$ & $6.41 \pm 0.65$ & $11.86 \pm 1.10$ \\
\hline
\end{tabular}


A

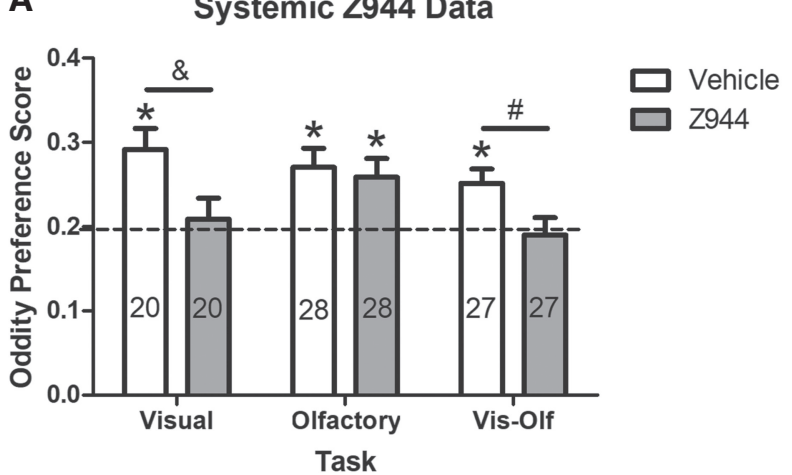

B

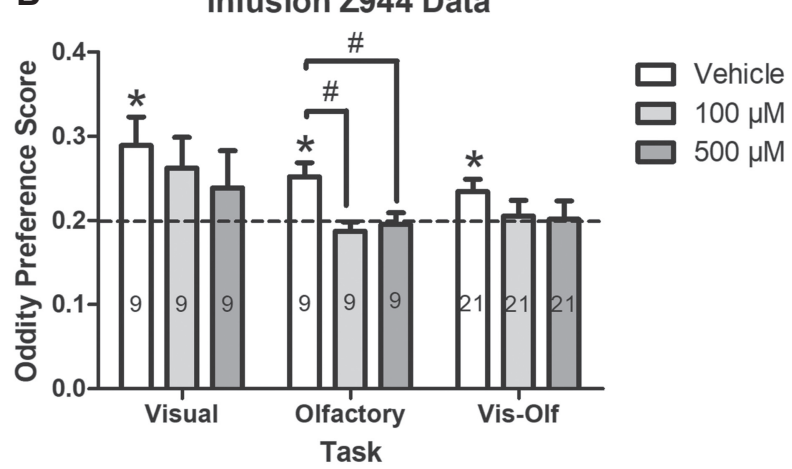

Figure 2. Visual, olfactory, and visual olfactory-oddity preference for systemic Z944 and orbitofrontal infusion Z944 treated rats. Significant impairments in oddity preference were found in systemic $Z 944$ treated rats in the visual $(\& P<0.05 ; A)$ and visual-olfactory (\#P<0.05; $A)$ tasks. Significant oddity preference above chance performance was found in vehicle treated rats for all task variants, and in Z944 treated rats in the olfactory task ( $\left.{ }^{*}<0.05 ; A\right)$. Significant impairments in oddity preference were found for the 100 and $500 \mu \mathrm{M} Z 944$ infusion doses during the olfactory task (\#P<0.05; $B$ ). Significant oddity preference above chance performance was found in vehicle infused rats for all task variants ( $\left.{ }^{\star} P<0.05 ; B\right)$.

of the visual features of the task (Bartko et al. 2007a,b). This evidence further supports the possibility that the PRh may be particularly sensitive to the systemic effects of Z944.

\section{Infusion of Z944 into the OFC disrupts uni- and multimodal MSO task performance}

Direct infusion of Z944 into the OFC produced a robust deficit in unimodal olfactory MSO performance regardless of dose. Impairments in unimodal visual and multimodal visual-olfactory performance were also demonstrated through nonsignificant performance of the task variants compared to chance for both doses of Z944. It should be noted that fewer rats were tested in the unimodal tasks than the multimodal task in an effort to reduce the number of infusions each animal sustained (see Materials and Methods for details). Thus, results of the unimodal tasks should be interpreted with caution given reduced power. We did not predict impaired unimodal visual task performance as previous researchers have shown intact visual CMOR performance following lesions to the OFC (Reid et al. 2014). In a separate study, lesions of the OFC were shown to selectively impair a tactile-olfactory configural learning task while olfactory processing remained intact (Whishaw et al. 1992). Although the OFC was not involved in these specific unimodal tasks, as discussed above the uni- and multimodal MSO tasks are unique in that they rely on the conjunctive representation of sensory features to successfully identify the odd object (Bartko et al. 2007b; Cloke et al. 2016). Thus, integration of stimulus features (whether they involve the same modality or different modalities) is a common feature of the task variants used in the present study. While it is tempting to propose that feature integration is the common function of the OFC in all three tasks, the MSO task does not lend itself to directly testing this hypothesis since the stimuli in all variants of the task must have at least two features (be they both from the same modality or different modalities). Others have shown involvement of the lateral OFC in odor discrimination tasks such as a go, no-go task that is more cognitively demanding than a simple discrimination (Eichenbaum et al. 1983). Interestingly, infusion of Z944 impaired olfactory MSO whereas systemic treatment did not. This pattern of results could potentially be explained by the variations in Z944 concentration in the OFC following systemic treatment versus direct infusion. Considering we did not directly measure T-type calcium channel activity in the OFC, this explanation is strictly speculative. However, the role of the OFC in olfactory-dependent cognition has been well documented (Slotnick 2001; Rolls 2004). In light of the impairments the in the unimodal visual and olfactory MSO tasks produced by Z944, future research should determine whether temporary lesions to the OFC also disrupt the unimodal MSO task variants.

The OFC has also been implicated in memory tasks dependent on MSI. In humans, enhanced OFC fMRI activity was found during an MSI task that involved the facilitation of odor detection by the simultaneous presentation of visually congruent cues. Importantly, increased OFC activation was unique to MSI trials and could not be explained by the summed activation of unimodal visual and olfactory stimulations (Gottfried and Dolan 2003). In a similar fMRI study, activity in the OFC increased progressively when a color-odor combination increased in consistency (e.g., yellow-lemon versus red-lemon) (Osterbauer et al. 2005). In rats, subchronic ketamine-induced impairments in visual-tactile and visual-olfactory MSI are reversed by OFC infusion of nicotine or the selective $\alpha_{4} \beta_{2}$ nicotine acetylcholine receptor agonist, ABT-418 (Cloke and Winters 2015; Cloke et al. 2016). The results from the current study further suggest that the OFC and, in particular, T-type calcium channel activity within the OFC mediate MSI. While the mechanism underlying this effect is unknown, one consequence of blocking T-type calcium channels would likely be to inhibit burst firing. Pyramidal neurons in OFC fire a portion of their action potentials in bursts and the number of bursts is affected by experience (Homayoun and Moghaddam 2006). In addition, T-type calcium channels may be involved in propagation of information from the thalamus to OFC (Deleuze et al. 2012), which may explain MSO task performance. Importantly, the function of the OFC is still a matter of considerable debate (Stalnaker et al. 2015). A recent proposal posits the OFC supports a "cognitive map" that defines task space (Wilson et al. 2014). Thus, computations performed in the OFC on incoming sensory information necessary for SI may contribute to the formation of a cognitive map. While these possibilities are speculative, they could be tested in future experiments.

\section{Conclusions}

The present study demonstrates the overall validity of the MSO, a paradigm which provides researchers the opportunity to study SI directly in rodents, as it has no mnemonic requirement. Our experiments also support previous work suggesting T-type calcium channel activity is required for normal SI and extend it to include 
the OFC as critical for SI. Additional research is required to substantiate these conclusions. In particular, because it was only recently developed, the construct validity of the MSO requires further examination. This could be accomplished by testing whether this assay is sensitive to the SI deficits previously reported in established rodent models of schizophrenia, epilepsy, and autism (Jacklin et al. 2012; Ballendine et al. 2015; Cloke and Winters 2015; Marks et al. 2016a; Siemann et al. 2017). Moreover, replicating the present study in these models would prove particularly fruitful in elucidating further the contribution of T-type calcium channel to MSI in the context of neuropsychiatric illness and in defining these channels as therapeutic targets.

\section{Materials and Methods}

\section{Animals}

Adult male Long Evans rats (Charles River Laboratories) were pair or single housed (pair housed if receiving systemic treatment, singly housed if receiving infusions) in standard polypropylene cages in a temperature controlled $\left(21^{\circ} \mathrm{C}\right)$ room with ad libitum access to food (Purina Rat Chow) and water. At the start of the experiment, rats ranged in age from 2-3 mo and weighed 300-350 g prior to when experiments were initiated. Behavioral testing was conducted during the light phase of a $12 \mathrm{~h}$ light-dark cycle (lights on at 07:00 h). All procedures adhered to the guidelines of the Canadian Council on Animal Care and were approved by the University of Saskatchewan's Animal Research Ethics Board.

\section{Drug and drug preparation}

A comprehensive overview of the synthesis and characterization of Z944 has been previously published. Previous research indicates Z944 is potent and selective, with inhibition of hCav3.1, hCAv3.2, and hCav3.3 T-type calcium channel isoforms occurring in the submicromolar range (Tringham et al. 2012).

\section{Systemic experiment}

On each test day Z944 was prepared fresh in a $1 \mathrm{mg} / \mathrm{mL}$ solution of $90 \%$ sodium carboxylmethyl cellulose (0.5\% saline, Sigma Aldrich) and 10\% dimethyl sulfoxide (DMSO; Sigma Aldrich). Z944 was administered intraperitoneally at a volume of $5 \mathrm{~mL} / \mathrm{kg}$, yielding a dose of $5 \mathrm{mg} / \mathrm{kg}$. This dose was selected based on previous research demonstrating that NEC animals display decreases in cross-modal memory performance following intraperitoneal administration of $\mathrm{Z} 944$ at $3 \mathrm{mg} / \mathrm{kg}$, whereas reduced locomotion occurs at $10 \mathrm{mg} / \mathrm{kg}$ (Marks et al. 2016a). Vehicle or Z944 was administered 15 min prior to MSO testing.

\section{Infusion experiment}

Stock solutions of Z944 were prepared in saline to yield concentrations of 100 and $500 \mu \mathrm{M}$. These stock solutions were kept refrigerated at $4^{\circ} \mathrm{C}$ and used throughout the course of testing. To our knowledge, Z944 has not been administered via this route to rats previously. For this reason, we selected molarities which exceeded the concentration shown to stop burst firing of in vitro thalamic preparations minimally by a factor of 10 , to account for dilution by cerebrospinal fluid in vivo (Tringham et al. 2012). During the infusion experiment, rats were gently restrained to facilitate the placement of infusion cannulae over each guide cannula. One $10 \mu \mathrm{L}$ Hamilton syringe was then connected to each infuser cannulae via propylene tubing for the delivery of the simultaneous bilateral OFC infusions. Under the control of a Harvard Apparatus syringe pump, each syringe dispensed a total of $1 \mu \mathrm{L}$ of vehicle or Z944 at a rate of $0.5 \mu \mathrm{L} / \mathrm{min}, 15 \mathrm{~min}$ prior to MSO testing. Following the infusion, the infusion cannulae were retained within the guide cannulae for $1 \mathrm{~min}$ to allow full diffusion. Prior to the infusion experiment, animals received two habituation sessions in which all aspects of the infusion procedure were carried out, with the exception of drug or vehicle administration.

\section{Surgery}

Surgery was performed on rats for the intracranial infusion experiment following previously reported methods (Cloke et al. 2016). Prior to and during the procedure, rats were anesthetized with the inhalant anesthetic isoflurane (Janssen). Preoperatively, all rats were administered a $0.5 \mathrm{mg} / \mathrm{kg}$ subcutaneous dose of the analgesic anafen (Merial Canada Inc). After animals were positioned in the stereotaxic apparatus, the scalp was cut and retracted to expose the skull. Holes were drilled and guide cannula were inserted bilaterally at the following coordinates corresponding to the ventrolateral (VL) OFC: anteroposterior (AP) $+3.65 \mathrm{~mm}$; lateral (L) $2.30 \mathrm{~mm}$; dorsoventral (DV) $-4.40 \mathrm{~mm}$ relative to bregma. To prevent obstruction, obdurators were placed into the cannulae, which were secured using six jeweler screws and dental acrylic before the scalp was sutured. Rats were singly housed post-operatively and recovered for 1 wk prior to behavioral testing.

\section{Apparatus and testing materials}

Habituation and testing occurred in a $60 \mathrm{~cm} L \times 60 \mathrm{~cm} \mathrm{W \times 60 \textrm {cm }}$ $H$ open field apparatus constructed from white corrugated plastic. The testing stimuli were created using $250 \mathrm{~mL}$ glass canning jars filled with autoclaved sand for stability. Three variations of the MSO task were used in our experiments: unimodal visual, unimodal olfactory, and multisensory visual-olfactory. Each task was composed of five objects, consisting of two pairs and an odd object that contained one feature from each pair (i.e., $\mathrm{AB}$ $\mathrm{AB} A D \mathrm{CD} C D$; Fig. 3). Objects for the unisensory and multisensory tasks comprised two distinct features from the same or different modalities, respectively. For the visual features, stickers of different colors, sizes, and shapes were affixed to laminated paper (Fig. 3A). For the olfactory task, spices $(25 \mathrm{~mL})$ were contained within lidless $50 \mathrm{~mL}$ plastic canisters, placed inside the glass jars to create distinct olfactory features (Fig. 3B). Holes were drilled in the jar lids of the olfactory, and visual-olfactory objects to enable scent sampling during testing. Jars and the testing apparatus were affixed with Velcro to prevent jars from tipping over during exploration.

\section{MSO testing protocol}

Rats were handled $5 \mathrm{~min} /$ day for a minimum of $3 \mathrm{~d}$ prior to testing. Rats were habituated for $5 \mathrm{~min} /$ day for two consecutive days prior to MSO testing. With the exception of the habituation sessions, behavior was recorded using an overhead video camera. Recordings of the testing sessions were manually scored using stop-watches, by a researcher blind to the treatment conditions. For each task, the five objects were presented simultaneously along one wall of the testing apparatus. Rats were given $5 \mathrm{~min}$ to explore the objects. The testing apparatus and stimuli were cleaned with $40 \%$ ethanol between subjects. The systemic and infusion experiments followed a within subjects' design, in which consecutive injections or infusions were minimally separated by a $48 \mathrm{~h}$ washout period. For the systemic and infusion experiments, each rat received six testing trials counterbalanced for drug treatment. All six testing sessions for each rat occurred over a 2 -wk period. For all experiments, task order, treatment, and odd object position were counterbalanced across rats and trials (i.e., the odd object appeared in all five possible positions for each rat throughout testing, and task variant and treatment were equally distributed throughout testing days).

Initially, a cohort of treatment naïve rats $(n=8)$ was run for the visual, olfactory, and visual-olfactory task variants (1 task/ day). Each rat was tested in all three task variants. For the systemic experiments, $(n=28)$, rats received either vehicle or Z944 treatment prior to the visual, olfactory, and visual-olfactory tasks (i.e., visual with vehicle, visual with Z944, olfactory with vehicle, olfactory with Z944, visual-olfactory with vehicle, visual-olfactory with Z944). Eight rats in the visual task were run using the wrong visual stimuli and were excluded from the visual task analysis. Thus, the final number of subjects for each task are: $n=20$ for the visual task, $n=28$ for the olfactory task, and $n=28$ for the visual-olfactory task. For the infusion experiments, two separate cohorts of rats were run to reduce the number of infusions received by each rat to six infusions. One set of rats, $(n=10)$ received vehicle, $100 \mu \mathrm{M}$ 

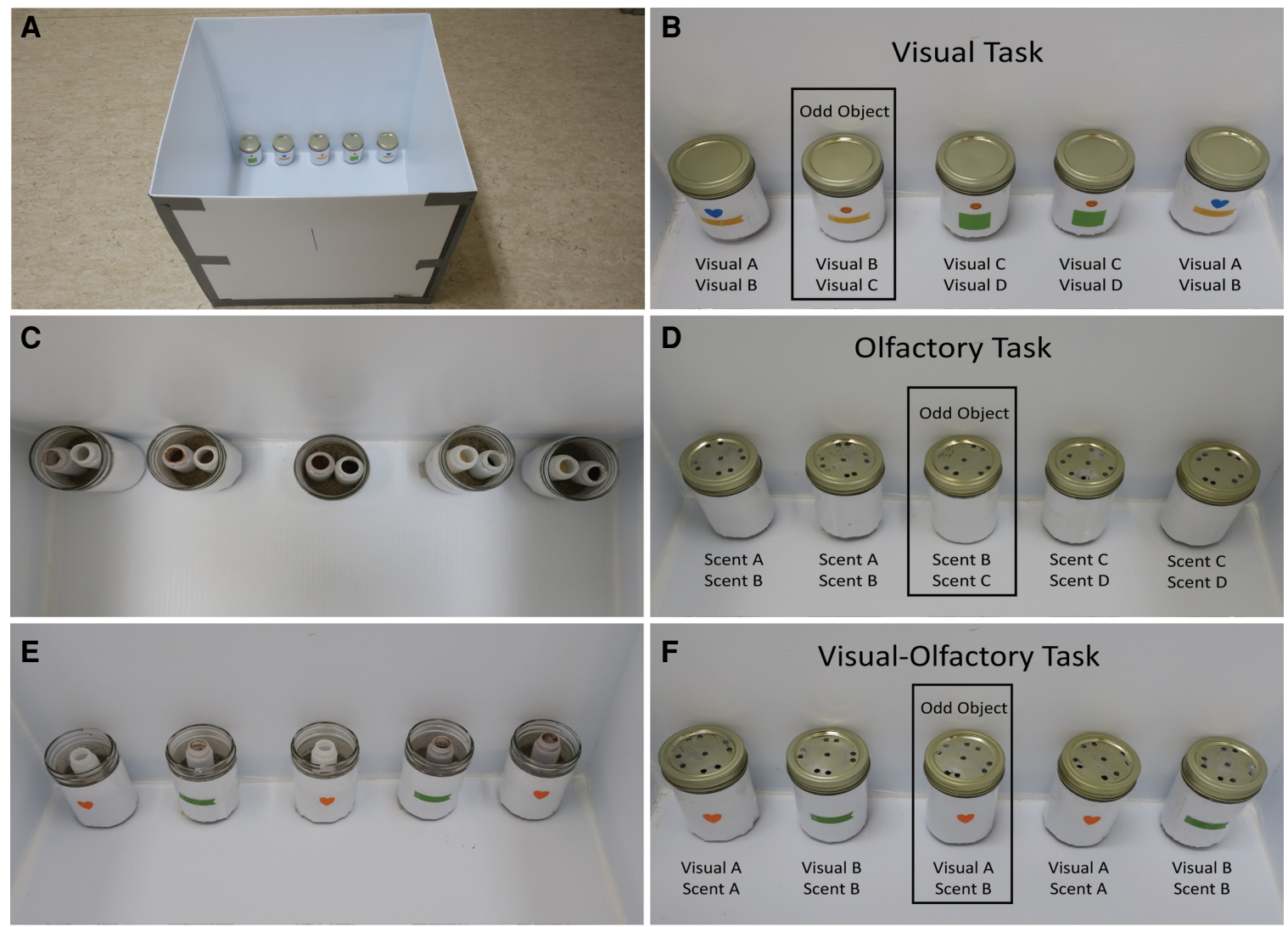

Figure 3. Photographs of the open field used for the MSO task $(A)$ and representative stimuli used for each task variant ( $B-F)$. Note that panels $C$ and $E$ show the stimuli with the lids off so the location of the vials containing the odors can be seen. Rats were always tested using stimuli similar to those shown in panels $B, D$, and $F$.

Z944, or $500 \mu \mathrm{M}$ Z944 for the visual and visual-olfactory tasks (i.e., visual with vehicle, visual with $100 \mu \mathrm{M} Z 944$, visual with $500 \mu \mathrm{M}$ Z944, visual-olfactory with vehicle, visual-olfactory with $100 \mu \mathrm{M}$ Z944, visual-olfactory with $500 \mu \mathrm{M}$ Z944). One rat was removed from the analysis for improper cannula placement (Fig. 4). A second set of rats, $(n=17)$ received vehicle, $100 \mu \mathrm{M} Z 944$, or 500 $\mu \mathrm{M}$ Z944 for the olfactory and visual-olfactory tasks (i.e., olfactory with vehicle, olfactory with $100 \mu \mathrm{M}$ Z944, olfactory with $500 \mu \mathrm{M}$ Z944, visual-olfactory with vehicle, visual-olfactory with $100 \mu \mathrm{M} \mathrm{Z944,} \mathrm{visual-olfactory} \mathrm{with} 500 \mu \mathrm{M}$ Z944). Five rats were removed from the analysis for improper cannula placement (Fig. 4). Also, three rats were removed from the olfactory analysis as they did not receive both doses of Z944 to complete the within subjects' design. Thus, the final number of subjects for each task are: $n=9$ for the visual task, $n=9$ for the olfactory task, and $n=21$ for the visual-olfactory task ( $n$ 's for each set were combined for the visual-olfactory task).

After MSO testing, rats from the infusion experiment were sacrificed with isoflurane and transcardially perfused with saline and then 4\% paraformaldehyde (Electron Microscopy Sciences). Brains were removed and post-fixed in paraformaldehyde before being transferred to a $30 \%$ sucrose solution and sectioned on a sliding microtome. Cannulae placement and infusion sites were confirmed using standard methods with reference to a rat brain atlas (Paxinos and Watson 2006).

\section{Data analysis}

The data were analyzed using the Statistical Package for the Social Sciences (SPSS) version 20 for Windows (IBM) with statistical significance set at $P \leq 0.05$ for all comparisons. Greenhouse-
Geisser corrections were used for instances of violation of sphericity (Mauchley's Test) for all repeated measures analysis of variance (ANOVA). Object exploration was quantified using an oddity preference score, which was calculated using the following formula:

Oddity Preference $=\frac{\text { time spent exploring the odd object }}{\text { total object exploration time }}$.

Interaction with either the olfactory or visual cues was considered to have occurred when the rat was sniffing the top of the jar lids within $2 \mathrm{~cm}$, or directed its visual gaze to the front semicircle of the jars within $2 \mathrm{~cm}$, respectively. For the systemic and infusion experiments, performance was analyzed using a repeated measures analysis of variance (ANOVA) to examine the effects of treatment and time bin on task performance. For the infusion experiments, post-hoc analyses were performed using simple effects' contrasts with a Bonferroni correction when required. To examine MSO task performance in each experiment, one-sample $t$-tests were used to compare group means to chance. Effect size was calculated using partial $\eta^{2}$ which represents the total variability in each dependent variable attributable to the independent variables. Partial $\eta^{2}$ values of $0.01,0.06$, and 0.14 are considered small, medium and large effect sizes, although these estimates should be interpreted with caution as little prior evidence of expected effects size is available for the MSO task (Cohen 1988). In all figures, the dashed line represents the oddity preference corresponding to chance. Oddity preference is reported as group means with error bars representing standard error mean (SEM). 


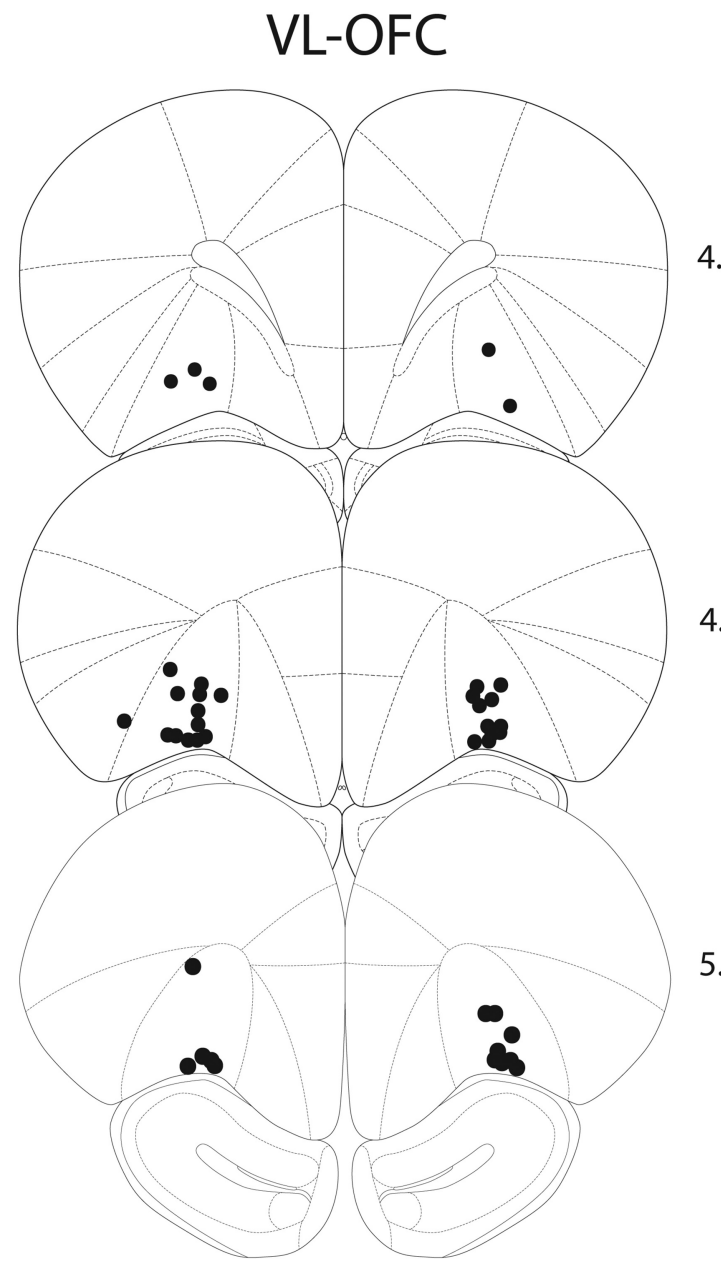

$4.20 \mathrm{~mm}$

$4.68 \mathrm{~mm}$

$5.16 \mathrm{~mm}$

Figure 4. Placements of the injections of Z944 into OFC. Guide cannula were inserted bilaterally corresponding to the $(\mathrm{VL})$ ventrolateral, (OFC) orbitofrontal cortex. Black dots indicate infusion sites confirmed from brains post-fixed in paraformaldehyde before being transferred to a $30 \%$ sucrose solution and sectioned on a sliding microtome.

\section{Acknowledgments}

This work was supported by a Brain Canada Multilnvestigator Research Initiative Grant with matching support from Genome British Columbia, the Michael Smith Foundation for Health Research, and the Koerner Foundation to T.P.S. Work in the laboratory of T.P.S. is supported by an operating grant from the Canadian Institutes for Health Research (CIHR; \#10677) and the Canada Research Chair in Biotechnology and GenomicsNeurobiology. Work in the laboratory of J.G.H. is supported by an operating grant from CIHR (\#125984), NSERC Discovery Grant, and matching funds for these experiments from the Saskatchewan Health Research Foundation and the University of Saskatchewan. W.N.M. is supported by funding from the Saskatchewan Health Research Foundation.

\section{References}

Andrade A, Hope J, Allen A, Yorgan V, Lipscombe D, Pan JQ. 2016. A rare schizophrenia risk variant of CACNA1I disrupts CaV3.3 channel activity. Sci Rep 6: 34233.

Ballendine SA, Greba Q, Dawicki W, Zhang X, Gordon JR, Howland JG. 2015. Behavioral alterations in rat offspring following maternal immune activation and ELR-CXC chemokine receptor antagonism during pregnancy: implications for neurodevelopmental psychiatric disorders. Prog Neuropsychopharmacol Biol Psychiatry 57: 155-165.
Bartko SJ, Winters BD, Cowell RA, Saksida LM, Bussey TJ. 2007a. Perceptual functions of perirhinal cortex in rats: zero-delay object recognition and simultaneous oddity discriminations. J Neurosci 27: 2548-2559.

Bartko SJ, Winters BD, Cowell RA, Saksida LM, Bussey TJ. 2007b. Perirhinal cortex resolves feature ambiguity in configural object recognition and perceptual oddity tasks. Learn Mem 14: 821-832.

Baum SH, Stevenson RA, Wallace MT. 2015. Behavioral, perceptual, and neural alterations in sensory and multisensory function in autism spectrum disorder. Prog Neurobiol 134: 140-160.

Caplan R, Siddarth P, Stahl L, Lanphier E, Vona P, Gurbani S, Koh S, Sankar R, Shields WD. 2008. Childhood absence epilepsy: behavioral, cognitive, and linguistic comorbidities. Epilepsia 49: 1838-1846.

Carter O, Bennett D, Nash T, Arnold S, Brown L, Cai RY, Allan Z, Dluzniak A, McAnally K, Burr D, et al. 2017. Sensory integration deficits support a dimensional view of psychosis and are not limited to schizophrenia. Transl Psychiatry 7: e1118.

Cloke JM, Winters BD. 2015. $\alpha_{4} \beta_{2}$ Nicotinic receptor stimulation of the GABAergic system within the orbitofrontal cortex ameliorates the severe crossmodal object recognition impairment in ketamine-treated rats: implications for cognitive dysfunction in schizophrenia. Neuropharmacology 90: 42-52.

Cloke JM, Nguyen R, Chung BY, Wasserman DI, De Lisio S, Kim JC, Bailey CD, Winters BD. 2016. A novel multisensory integration task reveals robust deficits in rodent models of schizophrenia: converging evidence for remediation via nicotinic receptor stimulation of inhibitory transmission in the prefrontal cortex. J Neurosci 36: 12570-12585.

Cohen J. 1988. Statistical power analysis for the behavioral sciences, 2nd ed. Lawrence Earlbaum Associates Hillsdale, NJ.

Deleuze C, David F, Béhuret S, Sadoc G, Shin H-S, Uebele VN, Renger JJ, Lambert RC, Leresche N, Bal T. 2012. T-Type calcium channels consolidate tonic action potential output of thalamic neurons to neocortex. J Neurosci 32: 12228-12236.

DiCarlo JJ, Zoccolan D, Rust NC. 2012. How does the brain solve visual object recognition? Neuron 73: 415-434.

Eichenbaum H, Clegg RA, Feeley A. 1983. Reexamination of functional subdivisions of the rodent prefrontal cortex. Exp Neurol 79: 434-451.

Gottfried JA, Dolan RJ. 2003. The nose smells what the eye sees: crossmodal visual facilitation of human olfactory perception. Neuron 39: 375-386.

Homayoun H, Moghaddam B. 2006. Progression of cellular adaptations in medial prefrontal and orbitofrontal cortex in response to repeated amphetamine. J Neurosci 26: 8025-8039.

Jacklin DL, Goel A, Clementino KJ, Hall AW, Talpos JC, Winters BD. 2012. Severe cross-modal object recognition deficits in rats treated sub-chronically with NMDA receptor antagonists are reversed by systemic nicotine: implications for abnormal multisensory integration in schizophrenia. Neuropsychopharmacology 37: 2322-2331.

Jones NC, Salzberg MR, Kumar G, Couper A, Morris MJ, O'Brien TJ. 2008. Elevated anxiety and depressive-like behavior in a rat model of genetic generalized epilepsy suggesting common causation. Exp Neurol 209: 254-260.

Jones NC, Martin S, Megatia I, Hakami T, Salzberg MR, Pinault D, Morris MJ, O'Brien TJ, van den Buuse M. 2010. A genetic epilepsy rat model displays endophenotypes of psychosis. Neurobiol Dis 39: 116-125.

Keefe RS, Harvey PD. 2012. Cognitive impairment in schizophrenia. Handb Exp Pharmacol 213: $11-37$.

Leekam S. 2016. Social cognitive impairment and autism: what are we trying to explain? Philos Trans R Soc Lond B Biol Sci 371: 20150082.

Marks WN, Cain SM, Snutch TP, Howland JG. 2016a. The T-type calcium channel antagonist Z944 rescues impairments in crossmodal and visual recognition memory in Genetic Absence Epilepsy Rats from Strasbourg. Neurobiol Dis 94: 106-115.

Marks WN, Cavanagh ME, Greba Q, Cain SM, Snutch TP, Howland JG. 2016b. The GAERS model of absence epilepsy exhibits alterations in fear conditioning and latent inhibition consistent with psychiatric comorbidities in humans. Eur J Neurosci 43: 25-40.

Oberman LM, Ramachandran VS. 2008. Preliminary evidence for deficits in multisensory integration in autism spectrum disorders: the mirror neuron hypothesis. Soc Neurosci 3: 348-355.

Ongür D, Price JL. 2000. The organization of networks within the orbital and medial prefrontal cortex of rats, monkeys and humans. Cereb Cortex 10: $206-219$.

Osterbauer RA, Matthews PM, Jenkinson M, Beckmann CF, Hansen PC, Calvert GA. 2005. Color of scents: chromatic stimuli modulate odor responses in the human brain. J Neurophysiol 93: 3434-3441.

Paxinos G, Watson C. 2006. The rat brain in stereotaxic coordinates, 6 th ed. Academic Press, New York, NY.

Powell KL, Cain SM, Ng C, Sirdesai S, David LS, Kyi M, Garcia E, Tyson JR, Reid CA, Bahlo M, et al. 2009. A Cav3.2 T-type calcium channel point mutation has splice-variant-specific effects on function and segregates with seizure expression in a polygenic rat model of absence epilepsy. J Neurosci 29: 371-380. 
T-type calcium cannels mediate sensory integration

Reid JM, Jacklin DL, Winters BD. 2014. Delineating prefrontal cortex region contributions to crossmodal object recognition in rats. Cereb Cortex 24 2108-2119.

Rolls ET. 2004. The functions of the orbitofrontal cortex. Brain Cogn 55: 11-29.

Siemann JK, Muller CL, Forsberg CG, Blakely RD, Veenstra-VanderWeele J, Wallace MT. 2017. An autism-associated serotonin transporter variant disrupts multisensory processing. Transl Psychiatry 7: e1067.

Slotnick B. 2001. Animal cognition and the rat olfactory system. Trends Cogn Sci 5: 216-222.

Splawski I, Yoo DS, Stotz SC, Cherry A, Clapham DE, Keating MT. 2006. CACNA1H mutations in autism spectrum disorders. J Biol Chem 281: 22085-22091.

Stalnaker TA, Cooch NK, Schoenbaum G. 2015. What the orbitofrontal cortex does not do. Nat Neurosci 18: $620-627$.

Stein BE, Stanford TR. 2008. Multisensory integration: current issues from the perspective of the single neuron. Nat Rev Neurosci 9: 255-266.

Talley EM, Cribbs LL, Lee JH, Daud A, Perez-Reyes E, Bayliss DA. 1999. Differential distribution of three members of a gene family encoding low voltage-activated (T-type) calcium channels. J Neurosci 19: 1895-1911.

Tamminga CA, Buchanan RW, Gold JM. 1998. The role of negative symptoms and cognitive dysfunction in schizophrenia outcome. Int Clin Psychopharmacol 13(Suppl 3): S21-S26.
Tringham E, Powell KL, Cain SM, Kuplast K, Mezeyova J, Weerapura M, Eduljee C, Jiang X, Smith P, Morrison JL, et al. 2012. T-type calcium channel blockers that attenuate thalamic burst firing and suppress absence seizures. Sci Transl Med 4: 121ra19.

Tseng HH, Bossong MG, Modinos G, Chen KM, McGuire P, Allen P. 2015. A systematic review of multisensory cognitive-affective integration in schizophrenia. Neurosci Biobehav Rev 55: 444-452.

Whishaw IQ, Tomie J, Kolb B. 1992. Ventrolateral prefrontal cortex lesions in rats impair the acquisition and retention of a tactile-olfactory configural task. Behav Neurosci 106: 597-603.

Wilson RC, Takahashi YK, Schoenbaum G, Niv Y. 2014. Orbitofrontal cortex as a cognitive map of task space. Neuron 81: 267-279.

Winters BD, Reid JM. 2010. A distributed cortical representation underlies crossmodal object recognition in rats. J Neurosci 30: 6253-6261.

Winters BD, Saksida LM, Bussey TJ. 2008. Object recognition memory: neurobiological mechanisms of encoding, consolidation and retrieval. Neurosci Biobehav Rev 32: 1055-1070.

Received January 15, 2018; accepted in revised form May 2, 2018. 


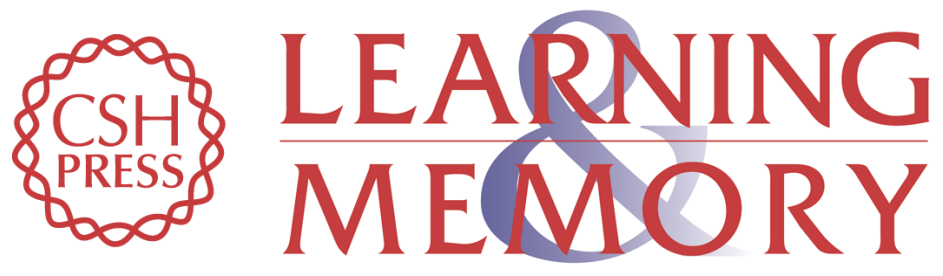

\section{T-type calcium channels in the orbitofrontal cortex mediate sensory integration as measured using a spontaneous oddity task in rats}

Wendie N. Marks, Madeline E. Parker, Nadine K. Zabder, et al.

Learn. Mem. 2018, 25:

Access the most recent version at doi:10.1101/Im.047332.118

References This article cites 40 articles, 10 of which can be accessed free at: http://learnmem.cshlp.org/content/25/7/317.full.html\#ref-list-1

Creative This article is distributed exclusively by Cold Spring Harbor Laboratory Press for the Commons first 12 months after the full-issue publication date (see

License http://learnmem.cshlp.org/site/misc/terms.xhtml). After 12 months, it is available under a Creative Commons License (Attribution-NonCommercial 4.0 International), as described at http://creativecommons.org/licenses/by-nc/4.0/.

Email Alerting Receive free email alerts when new articles cite this article - sign up in the box at the Service top right corner of the article or click here. 